\title{
LES VARIATIONS FRANÇAISES : RÉFLEXIONS D'ISIDORA SEKULIĆ SUR QUELQUES THEMMES ACTUELS DE LA LITTÉRATURE FRANÇAISE
}

Dans cet article, nous allons mettre en relief les essais d'Isidora Sekulić dans lesquels cette écrivaine évoque les auteurs français contemporains, à savoir ceux de la période entre-deux-guerres. Sur plus de trois cents essais qu'Isidora Sekulić a rédigés, soixante-quatorze concernent les littératures et les auteurs étrangers et parmi ceux-ci les écrivains et les critiques français occupent une place privilégiée. Par une analyse minutieuse et lucide de l'état actuel dans la littérature française ce qui signifie ni plus ni mois dans la littérature mondiale - Isidora Sekulić mène un dialogue intertextuel dynamique avec les poètes, les romanciers et les critiques français qui lui sont contemporains, tels Paul Valéry, l'abbé Bremond, Henri Massis, Paul Souday, Jean Giraudoux, Henri de Montherlant, Anatole France, André Malraux, y compris une contemporaine, Françoise Sagan, qui est la seule Française abordée par l'écrivaine serbe. Ceci dit, nous allons pointer les grandes lignes du dialogue prolifique tenu entre l'Est, représenté par notre Isidora Sekulić, et l'Ouest, représenté par de grands auteurs français de l'époque. Le but final est de montrer et d'affirmer la vive communication interculturelle qui existait entre ces deux cultures, grâce à l'activité littéraire et médiatrice d'Isidora Sekulić.

Mots-clés : Isidora Sekulić, intertextualité, interculturalité, essai, littérature française, critique littéraire française et serbe

Les contacts culturels font le bonheur des gens. La culture enrichit les gens, l'art fait le bonheur des gens, et les bonheurs sont internationaux, essentiellement humains depuis toujours. ${ }^{2}$

Isidora Sekulić

\footnotetext{
${ }^{1}$ Cet article est rédigé dans le cadre du projet scientifique Les langues, les littératures et les cultures romanes et slaves en contact et en divergence (no 81/1-17-8-01) soutenu par l'AUF (Agence universitaire de la Francophonie), l'Ambassade de France en Serbie et par le Ministère de l'éducation et du développement technologique.

${ }^{2}$ Tiré de l'essai Les contacts culturels font le bonheur des gens. Toutes les traductions des sources serbes sont les miennes.
} 


\section{Introduction}

Dans cet article, nous allons pointer certaines réflexions d'Isidora Sekulić (1877-1958) sur la littérature française contemporaine de son époque, à savoir, principalement de l'entre-deux-guerres. Isidora Sekulić est bien connue en tant qu'écrivaine et essayiste serbe, souvent plus appréciée comme essayiste dans le milieu littéraire serbe. Néanmoins, Slavko Leovac la reconnaît en tant que créatrice complète et bien formée dès son début littéraire: « Elle apparaît à la fois comme un subtil écrivain en prose, un lucide essayiste littéraire, un critique formé, traducteur de l'anglais, finalement comme journaliste au service du peuple » (LEOVAC 1977 : 8). Isidora Sekulić a rédigé plus de trois cents essais dont une vingtaine porte sur la France et les Français, à savoir sur l'apport spirituel et culturel de la France dans le monde entier, et au niveau plus universel, dans l'esprit de l'humanité. Avec une lucidité frappante Isidora Sekulić analyse tous les phénomènes actuels (souvent universels, de tous les temps) abordés dans l'œuvre littéraire de ses contemporains français tels Paul Valéry, l'abbé Bremond, Henri Massis, Paul Souday, Jean Giraudoux, Henri de Montherlant, Anatole France, André Malraux, Françoise Sagan. De ce fait, en rédigeant ses essais sur la littérature étrangère, Isidora Sekulić fait connaître au public lettré serbe l'état actuel dans la littérature française. Dans un premier temps, nous allons parler de sa vision des relations franco-serbes, et dans un deuxième temps nous allons souligner les grands thèmes de la littérature française contemporaine qu'Isidora Sekulić analyse et synthétise d'une façon magistrale.

Dans L'œuvre littéraire des femmes yougoslaves (1936 : 32), ouvrage de référence écrit en français, rédigé et édité par l'Association yougoslave des femmes diplômées des universités, il est dit d'Isidora Sekulić, à juste titre :

" Chacun de ses écrits, comme une synthèse de connaissances sans cesse accrues, de réflexion et d'expérience, marque un nouveau progrès sur la ligne de son développement. D’une immense culture, complétée par une excellente connaissance des langues depuis celles de l'antiquité, et des littératures étrangères, elle est aujourd'hui le juge le plus compétent et le plus autorisé en matière de littérature comparée. Ses récits de voyage, ses essais sur les auteurs yougoslaves et étrangers, ainsi que sur différents thèmes artistiques, ses réflexions sur des problèmes d'importance vitale, constituent à la fois la preuve de son goût artistique parfait, de sa profonde connaissance des choses, ainsi que de son sens philosophique et de sa conception du monde. »

Ce petit extrait témoigne vivement de la portée qu'aura Isidora Sekulić en matière de littérature et de culture serbes/yougoslaves et étrangères, notamment dans le domaine de l'essai dont la profondeur et l'épaisseur restent sans pareilles. 
Par définition, l'essai est « une énonciation en prose sur le sujet donné, dont l'objectif est choisi librement, et dont les moyens de composition et de style sont librement choisis par l'auteur qui y envisage ses expériences intimes, savoirs, pensées, réflexions, impressions et sentiments liés au sujet donné » (OSTROVSKI 2015 : 366). Ceci dit, ce genre littéraire se caractérise par une immense gamme thématique ainsi que par une importante flexibilité formelle. Il n'est donc pas surprenant que la disposition intellectuelle et émotionnelle d'Isidora Sekulić ait opté pour cette forme de réflexion profonde et dialogique, afin de communiquer avec les esprits de ses contemporains sur tous les méridiens, mais aussi, sur le plan universel, avec l'esprit de l'humanité entière.

Il est notoire que l'essai en tant que genre littéraire a été introduit par Michel de Montaigne qui a publié son seul ouvrage Essais en 1580 (première édition). Sous ce titre indicatif, Montaigne voulait pointer le fait de l'imperfection de l'être humain, du « moi » en évolution perpétuelle, de sorte qu'on ne peut qu'essayer et ressayer de l'examiner, c'est-à-dire de nous interroger sans cesse et sans prétendre à une solution, à une vérité définitive. Ou comme le formule l'auteur lui-même : « Il n'y a point de fin en nos inquisitions ; notre fin est en l'autre monde » (MONTAIGNE 1962 : 1045). Or, l'enjeu du terme « essai » est triple : d'abord c'est l'idée d' « essayer » une pensée, à savoir de la juger selon divers points de vue ; puis, « prendre une mesure, peser » (sens latin), à savoir s'essayer soi-même, tenter de soupeser sa vie, son expérience, ou celle des autres, de façon concrète ; finalement, au sens littéraire, l'essai est un genre privé de contrainte formelle, favorisant la liberté de réflexion et de jugement (ARMAND 1988 : 375-376). Certes, les essais d'Isidora Sekulić intègrent les trois sens cités y compris de nombreuses variations et transformations qu'un tel genre offre dans la liberté d' « essayer » à sa guise.

Dans une analyse comparative des pratiques essayistes de Francis Bacon et Montaigne, et en y comparant l'approche d'Isidora Sekulić, Ivanka Udovički arrive à la conclusion plus générale que l'essai d'Isidora est « un peu plus proche de Montaigne que de Bacon par une narration plus souple, par une expression artistique plus sophistiquée ainsi que par une expression plus relative des jugements sur l'homme, et surtout par le fait que les leçons ne sont pas exprimées ouvertement, mais ‘imprimées' dans le tissu de l'essai » (UDOVIČKI 1977 : 217). En plus, le travail essayiste du maître du genre et celui de l'essayiste serbe partagent certaines qualités essentielles, telles la procédure formelle et la stylisation, la compréhension artistique de l'essai, mais aussi le choix thématique (par exemple, les sujets de solitude, d'amitié, d'imagination, la puissance du raisonnement humain etc.).

Donc, Isidora Sekulić rédigeait ses essais librement, sans créer ni développer de système philosophique cohérent. Tout de même, elle revenait 
vers les questions fondamentales qu'elle considérait comme pertinentes en s'efforçant toujours de les résoudre à nouveau (RIBNIKAR 1986 : 320). Elle croyait à l'Art et à la Beauté : « [B] eauté de toute sorte, du son, de la couleur, de la forme, du mot. Ses essais sont à la recherche des valeurs artistiques : c'est l'analyse des valeurs plutôt que la définition. Essayiste jamais ennuyant, qui nous apprend en même temps l'art de vivre et la vie de l'art, Isidora Sekulić est un des écrivains rares vers qui on revient » (VELMAR JANKOVIĆ 1974 : 12). Or, pour cette occasion, nous allons nous attarder sur les essais qui concernent les grands auteurs français de l'époque et qui occupent l'attention de l'essayiste serbe.

2. «Eux, les Français, elles, les Françaises... »

Un essai tardif, datant de 1956, s'impose comme emblématique lorsqu'on parle de l'esprit français. Il est intitulé Eux, les Français, eux [Francuzi, oni, oni] - une litote qu'Isidora Sekulić emploie afin de nous rappeler le rôle exemplaire des Français qu'il faut adopter sans tarder. Ce sont eux, les Français, « les descendants les plus authentiques des anciens Grecs » dont les Serbes devraient suivre l'exemple. Malheureusement, Isidora Sekulić note avec amertume que les relations amicales franco-serbes sont fondées, selon « une inertie archétypale », sur l'histoire guerrière des Alliés, sur l'héroïsme sanglant, la période de l'occupation, sur la Mort en un mot. Même si l'on apprend la langue, la littérature et l'histoire culturelle françaises en Serbie, on n'arrive pas à fonder une école française, ce que les Turcs ont déjà fait en dehors des circonstances politiques. Et Isidora Sekulić se demande à juste titre : « Jusqu'à quand enfin, combien de temps encore incorporeronsnous le ciment sanglant dans tous les rapports? »(SEKULIĆ 1977a : 512).

À la différence du ton pessimiste du début de l'essai, Isidora Sekulić finit sur un ton optimiste lorsqu'elle reconnaît dans son pays et chez son peuple un vif sentiment selon lequel la parenté spirituelle avec la France doit être présentée comme un idéal : parmi toutes les vibrations de ce monde l'esprit français est le plus vibrant, il est en même temps poète et géomètre, il intègre idéalement l'esprit géométrique et l'esprit de finesse dont parlait Pascal, la science et les arts, les écrivains français sont législateurs et prophètes tel Jules Verne. Le Français est un bon cartésien en matière ainsi qu'en esprit, toujours mené par une méthode, loi, contrainte... Il est le seul à connaître la vraie mesure de liberté et de contrainte, et à l'instar de l'ancien Grec il connaît le vrai sentiment du Destin et de la condition humaine, André Malraux en étant le meilleur exemple (SEKULIĆ 1977a : 513-514, 517). Alors, Isidora Sekulić insiste surtout sur les valeurs immatérielles, intellectuelles et morales que 
l'esprit serbe devrait viser en idéal dans ses relations avec la grande culture française, car les contacts culturels font le bonheur des gens. C'est pourquoi elle plaide partout et toujours, de toutes ses forces, pour ce qu'on appelle aujourd'hui l'interculturalité, pour les échanges productifs et polyphones qui anoblissent le cœur et l'esprit, au lieu des guerres, du sang et de la mort qui les appauvrissent.

Après tout, il ne faut pas oublier « elles, les Françaises, elles ». Dans un essai controversé intitulé Constantin Bruner a-t-il le droit? de 1911 Isidora Sekulić rappelle une liste obligatoire des travaux des femmes sur le plan historique, en évoquant des représentants éminents de son sexe, dont, bien sûr, des écrivaines françaises telles Madame de Staël, George Sand et Louise Ackerman. Cependant, Isidora Sekulić considère leur contribution dans l'histoire comme « le début, le fondement, la pierre angulaire de l'accumulation difficile d'une énergie spirituelle et de la constitution d'une subjectivité » (KOCH 2012 : 264-265). Un essai tardif intitulé Françoise Sagan. Prix des critiques et davantage (1954) est entièrement dédié à une écrivaine française contemporaine, alors très jeune, âgée de dix-huit ans. Françoise Sagan a fait ses débuts littéraires en public avec le roman Bonjour, tristesse pour lequel elle a reçu le Prix des critiques en 1954. Isidora Sekulić, dans son âge mûr, en donne des réflexions tranchantes, n'épargnant ni les critiques, ni la jeune écrivaine.

Dans un premier temps, elle met en relief la critique masculine qui s'est trouvée confuse devant la tâche de juger une écrivaine " nouveau-née » aux qualités indubitables, malgré certains défauts. Cette critique reste indulgente envers l'œuvre d'une femme pourvu qu'elle soit jeune et belle, et si elle a un peu de talent - tant mieux ! Françoise Sagan est jeune, elle a du talent, mais elle n'est pas belle, selon la critique universitaire. Pourtant, Isidora Sekulić, suivant la lignée de l'esthétique romantique, trouve qu'il y a assez de charme dans sa tête "à la coupe de cheveux d'homme » et dans son visage « aux traits tranchants comme chez les petits rongeurs qui bougent sans cesse leur museau et dont les dents poussent constamment». De sa personnalité émane « un courage charmant, révélant que la flamme artistique n'est pas tout à fait céleste », mais, ajoutons-nous, grotesque. Pas belle - alors quoi ! Et, tout d'un coup, après ce petit plaidoyer combatif, Isidora Sekulić a brusquement pris conscience qu'elle tombait dans le même piège que ses collègues critiques : le piège du regard restreint concernant son sexe, à savoir le genre : «On dirait que ces lignes sont écrites par un jury féminin; il faut corriger » (SEKULIĆ $1977 b$ : 512-513).

Dans un deuxième temps, la correction est faite. L'essayiste serbe passe du cas spécifique de Françoise Sagan à un plan plus général - aux regards sur la littérature mondiale : car « votre cas est le nôtre aussi, parce que vous 
êtes Française ». Du coup, Isidora Sekulić y considère la scène littéraire mondiale par le biais de la France comme porte-bannière des littératures passant par une crise sévère : "La France a perdu la paix supérieure d'un être supérieur ». En effet, Isidora Sekulić craint l'hyperproduction de romans de tendances différentes, c'est-à-dire les aléas de la postmodernité. Un grand conflit éclate, la « guerre du livre » dans la littérature française. Dans ce contexte, l'écrivaine serbe semble reprendre l'idée et le message clés de son premier essai Constantin Bruner a-t-il le droit ? (donc, après 43 ans), et c'est que devant chaque talent, masculin ou féminin, serbe ou français, il y a un chemin difficile, laborieux et à long terme, chemin d'autoformation où il faut faire des efforts intellectuels et spirituels, car le vrai chemin n'est que celui qui mène per aspera ad astra. C'est pourquoi l'essayiste serbe estime qu'il est beaucoup plus nécessaire pour Françoise Sagan, après le coup de gloire, qu'elle s'attaque courageusement au « cauchemar » de la littérature française/ mondiale en crise, au lieu de la défendre de la rhétorique masculine à double face, à savoir des critiques français qui ont quand même reconnu son talent (SEKULIĆ 1977b : 514-515).

\section{La critique littéraire mise en cause}

À part la production littéraire, Isidora Sekulić s'intéresse bien évidemment aux problèmes des critiques et des talents critiques. Elle a consacré trois essais à trois critiques distingués de l'époque en France : ce sont Paul Souday, Henri Massis et l'abbé Bremond. Paul Souday, critique éminent et rédacteur en chef de la revue Temps dans les années 20, était un talent qui a manqué sa vraie vocation : une grande ambition, un grand travail, mais sec, dur, froid, infécond, un rationalisme violent mais privé d'imagination, sans don de modification ni de variation nécessaires dans les conclusions. Le plus grand défaut de sa critique est le manque de générosité, ce qui est impardonnable pour un esprit français. Souday aurait mieux fait d'avoir choisi la vocation de théologue érudit, professeur de latin, car il était justement ce type latin, scolastique, voué au scientisme et au scepticisme. Il n'a jamais pris conscience que « le critique doit absolument ressembler aux talents critiqués. Il possède les traits identiques, mais modifiés et variés à son tour de critique » (SEKULIĆ 1977c : 395-399).

Dans l'essai Un qui s'en doutait. Henri Massis Isidora Sekulić critique le pouvoir de nos capacités intuitives, où l'intuition est dirigée par l'inquiétude intérieure et l'angoisse. C'est le cas d'Henri Massis, académicien, critique et essayiste, patriote catholique, qui défend sans mesure l'Occident contre l'hérésie orientale, à savoir contre les prétentions culturelles, idéologiques et 
surtout politiques de l'Orient qui menace de dévorer l'Occident. Son inquiétude intérieure devant la désintégration de sa civilisation séculaire le mène à l'orientophobie qui ne défend plus les acquis occidentaux mais critique et juge l'Orient en tant que programme monstrueux. Dans sa peur existentielle qu'une nouvelle invasion des barbares dévasterait les valeurs de sa propre culture, Massis exagère en peignant l'Orient comme un "dragon apocalyptique ». C'est pourquoi il rêve d'une nouvelle Reconquista, il veut réhabiliter le catholicisme médiéval, non pas dans le sentiment religieux comme l'avait fait Chateaubriand autrefois, mais rénover l'organisation ecclésiastique, c'est-àdire le pouvoir de l'Église afin d'unifier l'Europe, assujettir l'Asie et établir finalement l'ordre et la stabilité voulus (SEKULIĆ 1977c : 384-388). Isidora Sekulić souligne le manque de conscience historique chez Massis, car il perd de vue le relativisme historique et national. Il méconnaît l'altérité des époques précédentes.

Dans son essai sur l'abbé Bremond, Isidora Sekulić touche la question de la poésie pure qui était alors actuelle et autour de laquelle s'est déclenchée une vive polémique publique, surtout entre Henri Bremond et Paul Valéry. Pour Bremond la poésie, comme la prière, est la plus haute extase de l'intellect humain, dont l'essence est purement mystique. En revanche, Valéry, que Bremond appelle « le poète malgré lui », tout en admettant la " fête de l'intellect », exigeait une discipline permanente dans la création, un souci d'exactitude presque hérétique, une rigueur perpétuelle, tandis que Bremond, en tant que religieux, plaidait pour l'intuition mystique et l'extase suprême. À la différence de Souday et Massis qui se perdent l'un dans son rationalisme cru, l'autre dans son intuition maladive, Bremond, érudit mais vivace, s'appuie sur l'intuition plutôt que sur la raison, en admirant ses résultats en tant qu'artiste et pas comme érudit. La raison, l'imagination, la sensibilité sont toujours des qualités impures, la seule chose entièrement pure c'est « l'expérience intérieure venue du contact mystique avec la plus haute réalité » qui reste ineffable. Valéry est aussi mystique mais notamment en matière de langage poétique, hérité directement de Mallarmé : sa langue est concise, son idée précise, et sa tête toute-puissante, à tel point qu'il n'a pas besoin de cœur (SEKULIĆ 1977c : 372-373, 377, 380).

\section{Le culte de l'Intellect}

Pour continuer avec Valéry, nous allons souligner qu'Isidora Sekulić a consacré deux essais à ce génie intellectuel : Paul Valéry à travers un souvenir et Paul Valéry - esquisse pour une étude, ce dernier étant inachevé et en même temps le plus long qu'Isidora Sekulić ait jamais écrit. Cela n'étonne 
pas vu que sa lucidité tranchante $"$ co-vibre $\|^{3}$ parfaitement avec l'intellect majestueux du génie français qu'elle admire. Les deux essais traitent des pouvoirs critiques de l'intellect, de ses capacités et de ses limites, à savoir des « efforts démoniaques » visant à percevoir de manière exhaustive le phénomène de la pensée humaine et son fonctionnement. Une tâche pénible mais sublime que Mallarmé nous a léguée. Dans cette veine de la littérature, Valéry semble être le dernier point. Les tendances abstraites de l'esprit sont idéalisées chez Valéry, son idéalisme est fondé sur les chiffres et les précisions mathématiques. Sa méthode est léonardienne " hostinato rigore, rigueur obstinée ». Heureusement, Valéry a su introduire l'âme et le cœur dans les abstractions froides - dans son récit poétique en prose, L'Âme et la danse, il a plongé cœur et tête dans sa poésie pure. "L'esquisse pour une étude » représente, entre autres, une minutieuse analyse comparative où Isidora Sekulić met en relation le «drame intellectuel » de Valéry avec les grands penseurs et humanistes depuis Héraclite, puis Platon, Aristote, Lucrèce, Saint-Augustin, Pétrarque, Dante, Shakespeare jusqu'à Baudelaire, Guérin, Proust, Tolstoï, Ibsen et Schopenhauer ! Dans ce parcours gigantesque, Isidora Sekulić finit par conclure qu'à la différence des auteurs cités ici, dont toutes les pensées abstraites amenaient à quelque chose de concret, chez Valéry les tendances abstraites de l'esprit restent au-dessus de tout (SEKULIĆ 1977c : 507-508, 511, 519, 523, 533-535).

Finalement, nous pouvons constater que le penchant vers l'intellectualisme, la clairvoyance et l'intelligence, vers « le cerveau qui est obligé d'être assez cerveau », sont sans doute les affinités électives d'Isidora Sekulić et Paul Valéry, parce que les deux s'en servent brillamment et s'en enivrent immodérement.

\section{Approche comparative, approche anecdotique}

Comme nous venons de l'aborder, Isidora Sekulić a souvent recours à l'analyse comparative et à l'interprétation comparative des auteurs français. ${ }^{4}$ Ainsi, nous avons un essai intitulé Les variations françaises. Jean Giraudoux et Henri de Montherlant. Mais, il ne s'agit pas seulement d'une analyse purement littéraire, comme le dit Isidora Sekulić ; ces deux auteurs sont mis ensemble comme deux thèmes musicaux, essentiellement français pour une petite variation française. Donc, Isidora Sekulić songe à un syncrétisme

\footnotetext{
${ }^{3}$ Les termes co-vibrer et co-vibration sont forgés par Isidora même. Elle y entend une interaction harmonieuse et synergique des esprits subtils qui se partagent les affinités électives.

${ }^{4}$ Même les caractéristiques physiques et matérielles n'échappent pas à son œil de photographe, étant donné que le physique correspond souvent au psychique.
} 
artistique où Giraudoux et Montherlant correspondent textuellement à deux thèmes principaux d'une composition polyphonique. ${ }^{5}$ André Gide, en même temps qu'Isidora Sekulić, dans le « Journal d'Éduard» (1925) parle de cette analogie constructive: «Ce que je voudrais faire, comprenez-moi, c'est quelque chose qui serait comme l'Art de la fugue. Et je ne vois pas pourquoi ce qui fut possible en musique serait impossible en littérature » (GIDE 1991 : 187). De surcroît, Isidora Sekulić utilise la forme d'un essai littéraire non seulement comme moyen de dialogue intertextuel polyphonique, mais également comme moyen d'interpréter et d'intégrer d'autres pratiques culturelles et artistiques comme la musique, l'architecture, le ballet, le théâtre etc. Dans ce sens, il faut citer les essais comme La cathédrale de Chartres, Le jardin du Luxembourg, " Coppélia » de Délibes, Sarah Bernhardt.

Voici quelques lignes où Isidora Sekulić « compose » des variations typiques à la française : "Giraudoux et Montherlant, les deux préfèrent prendre soi-même pour le protagoniste de leur livre. Bien sûr, de manières très différentes. [...] Les personnalités de leurs romans et drames auraient été bien plus pauvres s'il n'y avait pas en eux, et sur eux, une multitude de lignes et de motifs cernés avec soin ». Les deux sont sportifs, mais Giraudoux en fait pour la santé et l'allégresse, et Montherlant pour les risques où il dépasse sans cesse les limites de son individualité. Les deux étaient en guerre, mais chacun en a tiré sa propre expérience. Giraudoux est un diplomate qui s'adonne à l'art d'ironiser et pointer, et pour être politique, il n'est jamais devenu poète : il manque de passion et d'héroïsme montherlanesque, la passion qu'il a consumée dans les Affaires étrangères. Montherlant lutte pour les idéaux aristocratiques, voire cornéliens, pour un être parfait, toujours « meilleur, plus fort, plus digne », visant un seul but jusqu'au bout, tandis que Giraudoux est « un fleuve héraclitéen qui change son cours », passant par les « vérités moyennes », contournant le pathétique, et finissant par l'ironie, le comique et le burlesque. Au final, « ce que Montherlant présente comme le joug moral de l'homme, Giraudoux le voit tout simplement comme ses fonctions » (SEKULIĆ 1977c : 408-409, 412-413, 416, 419).

Dans ses analyses comparatives et souvent biographiques - que la nouvelle critique réfutait et considérait comme dépassées - Isidora Sekulić, par une observation et association brillantes des faits « banals », savait parfaitement attirer l'attention d'un lecteur sensible pour le mener ensuite vers l'essence profonde. Certes, elle est pleinement consciente que la biographie

\footnotetext{
${ }^{5}$ Aujourd'hui, il est de notoriété que Julia Kristeva avait repris les termes « polyphone » et « polyphonique » pour désigner le dynamisme immanent au texte. Il s'agit du dialogue actif qui s'enchaîne à l'infini dans la production textuelle « travaillante ». Les textes s'entrelacent et se superposent simultanément tout comme dans une composition polyphonique à contrepoint de Jean-Sébastien Bach. Comme nous pouvons constater, cette idée n'était pas étrangère à Isidora Sekulić qui en réfléchissait déjà avant la révolution du langage poétique de Kristeva.
} 
crue ne mène pas vers les quintessences, mais on en a besoin de crainte de se perdre dans les abstractions froides. C'est pourquoi elle préfère ce que nous appelons une " approche anecdotique » qu'elle applique en analysant la vie et l'œuvre de Stendhal, Balzac, France et autres. Par exemple, dans l'essai Anatole France Isidora Sekulić note le fait que «France est un des ceux qui portent un bonnet drôle - il le portait vraiment - faisant peur aux gens ». Ensuite, elle nous rappelle la personnalité spécifique qu'était Dante et le compare avec France : " $\mathrm{Au}$ moment où Dante apparaissait avec son traditionnel foulard rouge, les rues florentines se vidaient aux cris d'enfants effrayés : fichez le camp! Voici l'homme au foulard rouge! Il vaut de même pour France, surtout après sa mort, alors après son nom, les gens s'écrient : allez, bougez ! Le voilà, l'homme à bonnet » (SEKULIĆ 1977b : 332, 339).

À notre sens, un tel procédé anecdotique n'est nullement superflu ni « banal ». Au contraire, il nous entraîne dans le mystère des figures littéraires et de la littérature elle-même de la façon la plus simple. L'anecdote anoblit les faits secs (parce que France portait vraiment un bonnet) et les recouvre d'une première couche de valeur littéraire - mince mais pas négligeable : une anecdote ou « histoire ", pourvu qu'elle soit habilement racontée, est un moyen d'entrer dans le vaste champ des essences littéraires à découvrir. Les faits en tant que tels ne sont pas toujours un motif artistique ; mais quand ils parcourent les souvenirs de ceux qui savent parler, et « ceux qui savent fabuler peuvent créer des mondes », quand ils traversent de tels souvenirs, ils deviennent universels et humains, des thèmes purement artistiques, et puis des souvenirs encore plus forts des générations postérieures (SEKULIĆ 1977a : 464). Dans une lettre à Barrault, Paul Claudel parle aussi du « saut qualitatif » du concret à l'universel, à savoir de ce passage du sentiment cru au sens, finalement de l'anecdote à la parabole (LIOURE 1998 : 54).

\section{Autres thèmes}

Outre les essais cités, on trouvera des références (évidentes ou sousjacentes) à la littérature française dispersées dans d'autres essais où Isidora Sekulić aborde les problèmes universels dans l'espace littéraire de son époque. Dans les Isochimènes en littératures l'essayiste serbe souligne le « talent pour la révision » que chaque génie moderne devrait avoir impérativement, tout révolutionnaire qu'il soit, car les modernes ne sont qu' « un retentissement d'une ancienne révolution inhérente à une plus grande et plus vieille substance » (SEKULIĆ 1977b : 48), c'est-à-dire à un intertexte précédent selon Barthes, ou à un hypotexte, voire architexte selon Genette. Ceci dit, 
l'isochimène ${ }^{6}$ isidorienne serait cette « ligne bleue » et imaginaire sur laquelle s'inscrivent les mêmes tendances littéraires, mais modifiées par l'altérité du contexte où le nouveau texte est né : « depuis Baudelaire, traversant Verlaine, Laforgue, Samain, Ghil et Vildrac - les plus modernes et les plus nouveaux sont le cinquième ou le sixième point sur la ligne, ou bien une réactualisation du vieux symbolisme » (SEKULIĆ 1977b : 54). Tel le jeune Cocteau qui a écrit son roman Le grand écart en étant absolument humain et universel, mais en même temps très moderne, " aux images curieusement fraîches et bien réussies ». Par conséquent, ces images nouvelles, « fraîches et réussies » viennent avec l'esprit du temps moderne, avec l'altérité historique qui peint l'isochimène d'une autre nuance de bleu.

Aux réflexions sur les révolutions, sur la modernité et la tradition en littérature, rajoutons les "solitudes ». L'essai Isolements en littératures examine le phénomène contemporain de "l'isolement 》 des écrivains, à savoir la disparition progressive de groupes littéraires qui étaient autrefois essentiels pour la vie et la pensée littéraires. C'était l'époque des cénacles où Charles Nodier rassemblait chez lui à Paris des écrivains et des artistes qui préparaient la révolution du romantisme, ou bien des parnassiens français qui se rassemblaient autour du Parnasse contemporain pour créer après un cercle littéraire symboliste. Isidora Sekulić discerne les écrivains solitaires d'après leur condition - le contexte politique et social, et ceux qui sont solitaires par leur choix, comme Romain Rolland et Marcel Proust qui optent volontairement pour la prose intimiste. Cette orientation chez Rolland et Proust était prodigieusement féconde en matière artistique. Contrairement à la critique littéraire actuelle, qui dans le manque de communautés littéraires voit le manque d'inspiration poétique, ce qui donnerait de grandes œuvres synthétiques, l'essayiste serbe cite de nombreux exemples qui renforcent le point de vue opposé : " dans l'éclatement des groupes et l'isolement des écrivains, quelque chose de positif peut être vu, issu de quelque chose de positif. [...] Les destinées de la solitude et les instincts de la solitude signifient guérison et consécration » (SEKULIĆ 1977b : 54).

\section{En guise de conclusion}

Tout compte fait, à la fin de notre parcours « français » des essais d'Isidora Sekulić, où elle aborde divers sujets littéraires, nous ne pouvons que constater une immense richesse d'esprit humain que le génie de l'essayiste serbe nous fait voir, comprendre et admirer finalement. Qu'elle parle du plus

\footnotetext{
${ }^{6}$ En géographie, la ligne isochimène est une ligne passant par tous les points de la terre qui ont la même température moyenne en hiver.
} 
authentique descendant des anciens Grecs, ou de l'esprit serbe qui devrait en prendre exemple, qu'elle pratique l'approche comparative ou anecdotique, qu'elle critique ou théorise sur la portée des critiques ou des écrivains bien connus et reconnus, quoi qu'elle fasse, sa plume reste conséquente et fidèle aux trois divinités capitales : Vérité, Beauté et Art. Elle est toujours sincère jusqu'au bout envers soi-même tout comme envers les autres et lorsqu'il faut dire la vérité, que ce soient les vertus ou les défauts, Isidora Sekulić n'épargne personne. Bien qu'elle partage avec Valéry les efforts intellectuels visant la plus haute vérité, elle n'estime pas moins les capacités du cœur, de l'intuition et de l'imagination qui sont d'ailleurs plus proches de l'Art. De ce fait, les textes analytiques d'Isidora Sekulić sont remplis non seulement d'une forte intellectualité valérienne, mais aussi d'une sensibilité subtile pour tout type de connaissance: imaginative et intuitive, empiriquement et discursivement, en extase philosophique ou religieuse, âme et corps, esprit et cœur : simplement « tout savoir par la pensée abstraite et avec du sang et de la chair », comme le dit elle-même à propos de La voie royale de Malraux.

Et pourtant, il ne reste à la fin que le texte écrit : c'est pourquoi Isidora Sekulić croit le plus à la beauté du mot qu'elle cisèle à perfection afin d'y imprimer ses réflexions approfondies. De plus, en lisant ses essais dignes de son grand prédécesseur Montaigne, le lecteur attentif « s'essaie » en sa propre pensée et en sa propre compréhension des choses : il travaille à bien penser, et selon Pascal, toute la dignité de l'homme est là. De ce fait, Isidora Sekulić privilégie le travail minutieux sur la forme, sur le style, sur l'expression précise, qui sont les valeurs classiques dont ses essais témoignent vivement. Car, seule une textualité bien composée peut trouver des échos et des réponses plus profonds dans le réseau intertextuel, tout comme une musique bien composée trouve immédiatement une réponse vibrante dans le cœur et l'esprit d'un auditeur attentif. Si Isidora Sekulić considère Giraudoux et Montherlant comme «d'excellents sujets pour une petite variation française », ses essais, que nous venons d'envisager, peuvent être considérés comme d'excellents sujets pour une grande fugue polyphonique française, et cette polyphonie, heureusement, touche le monde entier. C'est pourquoi l'essayiste serbe met en idéal les contacts culturels qui font « bonheur des gens » en invitant notre petite mais riche culture à s'insérer, sans tarder, dans cet espace fécond et infini qu'on appelle aujourd'hui le dialogue des cultures ou l'interculturalité.

\section{Bibliographie}

ARMAND 1988 : ARMAND, Anne. Moyen-Âge. XVI siècle. Collection Itinéraires littéraires. Paris : Hatier, 1988. 
KOCH 2012 : KOCH, Magdalena. ...kada sazremo kao kultura... Stvaralaštvo srpskih spisateljica na početku XX veka (kanon - žanr - rod) [... lorsqu'on mûrit en tant que culture... L'œuvre littéraire des écrivaines serbes au début du XX siècle. Canon-genre]. Beograd: Službeni glasnik, 2012. [orig.] КОХ, Магдалена. ...када сазремо као култура... Стваралаштво српских списатељица на почетку XX века (канон - жанр - род) [...au moment où l'on mûrit comme culture... L'ouvre littéraire des écrivaines serbes au début du XXe siècle. Canon-genre] Београд: Службени гласник, 2012.

LEOVAC 1977 : LEOVAC, Slavko. « Isidora Sekulić ». Kritički radovi Isidore Sekulić [Travaux critiques d'Isidora Sekulić]. Novi Sad-Beograd: Matica srpska, Institut za književnost i umetnost, 1977.

LIOURE 1998 : LIOURE, Michel. Lire le théâtre moderne de Claudel à Ionesco. Paris : Dunod, 1998.

L'EUVRE LITTÉRAIRE DES FEMMES YOUGOSLAVES 1936 : Rédigé par l'Association yougoslave des femmes diplomées des universités, Dubrovnik : Édition du Conseil national des Femmes yougoslaves, 1936.

OSTROVSKI 2015 : OSTROVSKI, Vitold. «Esej » [Essai]. Rečnik književnih rodova $i$ vrsta [Dictionnaire des genres littéraires]. Redakcija Gžegož Gazda i Slovinja Tinecka Makovska. Prevela s poljskog Ivana Đokić Saunderson. Beograd: Službeni glasnik, 366-370, 2015.

RIBNIKAR 1986 : RIBNIKAR, Vladislava. Književni pogledi Isidore Sekulić [Regards littéraires d'Isidora Sekulić]. Beograd: Prosveta, 1986.

UDOVIČKI 1977 : UDOVIČKI, Ivanka. Esej Isidore Sekulić [L'essai d'Isidora Sekulić]. Beograd: Institut za književnost i umetnost, 1977. [orig.] УДОВИЧКИ, Иванка. Есеј Исидоре Секулић. Београд: Институт за књижевност и уметност, 1977.

VELMAR JANKOVIĆ 1974 : VELMAR JANKOVIĆ, Svetlana. Isidora Sekulić. Beograd: Narodna knjiga, 1974.

\section{Sources}

GIDE 1991 : GIDE, André. Les Faux-monnayeurs. Paris : Gallimard, 1991.

MONTAIGNE 1962 : MONTAIGNE, Michel de. Essais. Livre III. Bibliothèque de la Pléiade. Paris : Gallimard, 1962.

SEKULIĆ 1977a : SEKULIĆ, Isidora. „Francuzi, oni, oni“ [Eux, les Français, eux]; „Kulturni dodiri sreća su ljudi“" [Les contacts culturels font le bonheur des gens]. Sabrana dela Isidore Sekulić [Euvres complètes d'Isidora Sekulić]. Govor i jezik - Mir i nemir [Parler et langue - paix et angoisse], knjiga 10. Beograd: Vuk Karadžić, 1977, 511-517, 460-478. [orig.] СЕКУЛИЋ, Исидора. „Французи, они, они“; „Културни додири срећа су људи“. Сабрана дела Исидоре Секулић. Говор и језик - Мир и немир, књига 10. Београд: Вук Караџић, 1977, 511-517, 460-478. 
SEKULIĆ 1977b : SEKULIĆ, Isidora. „Fransoaz Sagan, nagrada kritičara i dalje“ [Françoise Sagan, Prix des critiques et davantage]; „Anatol Frans“ [Anatole France]; „Izohimene u književnostima“ [Isochimènes en littératures]; „Samovanja u književnostima“ [Isolements en littératures]. Sabrana dela Isidore Sekulić. Iz stranih književnosti II [Des littératures étrangères II], knjiga 8. Beograd: Vuk Karadžić, 1977, 507-515, 331-346, 47-57, 58-71. [orig.] СЕКУЛИЋ, Исидора. „Франсоаз Саган, награда критичара; и даље“; „Анатол Франс“; „Изохимене у књижевностима“; „Самовања у књижевностима“. Сабрана дела Исидоре Секулић. Из страних књижевности II, књига 8. Београд: Вук Караџић, 1977, 507-515, 331-346, 47-57, 58-71.

SEKULIĆ 1977c : SEKULIĆ, Isidora. „Ka problemu kritičara. Pol Sude“ [Vers le problème des critiques. Paul Souday]; „Jedan koji je slutio. Anri Masi“ [Un qui s’en doutait. Henri Massis]; „Opat Bremon“ [L'abbé Bremond]; „Pol Valeri - skica za studiju“ [Paul Valéry - esquisse pour une étude]; „Francuske variacije. Žan Žirodu i Anri de Monterlan“ [Les variations françaises. Jean Giraudoux et Henri de Montherlant]. Sabrana dela Isidore Sekulić. Iz stranih književnosti I [Des littératures étrangères I], knjiga 7. Beograd: Vuk Karadžić, 1977, 395-405, 384-394, 371383, 507-567, 406-419. [orig.] СЕКУЛИЋ, Исидора. „Ка проблему критичара. Пол Суде“; „Један који је слутио. Анри Маси“; „Опат Бремон“; „Пол Валери - скица за студију“; „Француске вариације. Жан Жироду и Анри де Монтерлан“. Сабрана дела Исидоре Секулић, Из страних књижевности I, књига 7. Београд: Вук Караџић, 1977, 395-405, 384-394, 371-383, 507-567, 406-419.

\section{Владимир 3. Ђурић}

\section{ФРАНЦУСКЕ ВАРИЈАЦИЈЕ: РАЗМИШЉАҢА ИСИДОРЕ СЕКУЛИЋ О НЕКИМ АКТУЕЛНИМ ТЕМАМА У ФРАНЦУСКОЈ КЮИЖЕВНОСТИ}

У раду анализирамо оне есеје Исидоре Секулић у којима се она позива на тада савремене ауторе у француској књижевности, што значи на писце и књижевне критичаре који су стварали махом у првој половини ХХ века. Од више од три стотине есеја, колико их је Исидора Секулић написала, седамдесет и четири су из страних књижевности, а међу њима француски писци и критичари заузимају посебно место. Луцидном и минуциозном анализом актуелног стања у француској књижевности, Исидора Секулић води динамичан интертекстуални дијалог са савременим и водећим песницима, писцима и књижевним критичарима као што су: Пол Валери, опат 
Бремон, Анри Маси, Пол Суде, Жан Жироду, Анри де Монтерлан, Анатол Франс, Андре Малро, Франсоаз Саган и други. Ми ћемо се овде задржати на неким кључним местима, текстуалним и мисаоним чвориштима у радовима наведених аутора око којих се плете плодан дијалог између Истока, оличеног за ову прилику и сасвим оправдано у Исидори Секулић, и Запада, оличеног у списима француских великана. Главни циљ је да покажемо и потврдимо живу интеркултурну комуникацију која се одвијала у том периоду на нашем књижевном поднебљу, и то посебно захваљујући мисаоно продубљеној и активистичкој улози Исидоре Секулић.

Кључне речи: Исидора Секулић, интертекстуалност, интеркултуралност, есеј, француска књижевност, француска и српска књижевна критика 